\title{
Signal amplification by combining two advanced immunohistochemical techniques
}

\author{
D. Russo, A. Ambrosino, A. Vittoria, A. Cecio \\ Department of Biological Structures, Functions and Technology, University of Naples "Federico II", Italy
}

.

(C)2003, European Journal of Histochemistry

The immunohistochemical techniques known as EnVision ${ }^{\mathrm{TM}_{+}}$ System (EVS) and Mirror Image Complementary Antibody (MICA) were recently introduced into laboratory practice because of their high sensitivity. In this paper these techniques were compared and their sequences combined to obtain a new method possibly more sensitive than the original ones. The immunohistochemical staining employing the avidin-biotin complex $(A B C)$, largely used as routine, was adopted as a term of comparison.

Samples from the small and large intestine of pigs and sheep were fixed in Bouin and embedded in Paraplast. The primary antibodies utilized were directed against the neuronal nitric oxide synthase (nNOS), vasoactive intestinal polypeptide (VIP) and chromogranin A ( $\mathrm{Cr}$ A). Targets of these antibodies were nerve structures of the intestinal wall, as well as endocrine cells scattered in the mucosa of the bowel, defined neuroendocrine cells or paraneurons.

The EVS method appeared as slightly superior to the MICA method regarding sensitivity of detection. The EVS/MICA (combined) method resulted four/eight times more effective than the original techniques regarding sensitivity of detection and staining intensity, both at low and high dilutions of the primary antibodies. Of these latter, immunopositive structures were still clearly identifiable, at a dilution of $1: 256,000$. Such efficiency could be explained by the high number of revealing molecules of peroxidase contained in the new sequence. The application of the combined method is recommended when a small quantity of tissue antigens needs to be detected immunohistochemically.

Correspondence: Prof. A. Vittoria, Department of Biological Structures, Functions and Technology, University of Naples "Federico II", via Veterinaria 1, 80137 Naples, Italy.

Phone: international +39.081.2536099. Fax. international +39.081.2536097. E-mail: avittoria@unina.it

Key words: immunohistochemistry, EnVision ${ }^{\mathrm{TM}}+$ system, MICA method, EVS/MICA method, signal amplification.

Paper accepted on May 27, 2003

European Journal of Histochemistry 2003; vol. 47 issue 4 [0ct-Dec]: 379-384
7 he main objective of immunohistochemical research is the elaboration of new techniques having high sensitivity in tissue antigen revelation. Many of such techniques have been developed quite recently and for this reason they are still poorly known and rarely applied in current laboratory use. This is the case with the methods defined respectively as EnVision ${ }^{\mathrm{TM}_{+}}$System (EVS) and Mirror Image Complementary Antibody (MICA). The first (Heras et al. 1995, Bisgard and Pulzek 1996, Sabattini et al. 1998) is characterized by a long dextran molecule binding immunoglobulins (IgGs) as secondary antibodies and peroxidases as revealers; the second (Mangham and Isaacson 1999) is a multistep sequence forming a network of IgGs linked to tissue antigens by primary antibodies and containing peroxidases in its strands. Besides the said signal amplification these techniques allow high dilution of the primary antibodies, tight binding affinity between detecting antibodies and tissue antigens and lacking of endogenous biotin background because they do not utilize the avidin molecule. In this paper these techniques have been compared to each other as well as to the routine immunohistochemical method utilizing the avidinbiotin complex (ABC) (Hsu et al. 1981) which has been chosen as a reference term.

Combination of the sequences of two techniques or repetition of some steps of a multilayer method have also been used in the last two decades to obtain signal amplification in immunohistochemistry (Vacca et al. 1975, Swanson et al. 1987, Davidoff and Schulze 1990).

Principal aim of this work is to combine the sequences of the EVS and MICA methods in order to obtain a new immunohistochemical technique possibly more sensitive than both the originals.

\section{Materials and Methods}

The material used in this study consisted of samples of small and large intestine collected from 
three pigs and three sheep in a local slaughterhouse, soon after the death of the animals. The subjects were adult and of both sexes. The material was washed in a $0.01 \mathrm{M}$ phosphate buffered saline solution (PBS) ( $\mathrm{pH}$ 7.4) and fixed in Bouin's fluid overnight at room temperature (r.t.). Then it was routinely dehydrated, embedded in Paraplast and microtomically cut in $5 \mu \mathrm{m}$ thick sections. These latter were deparaffinized in xylene, rehydrated through serial alcohols and water, and washed in $0.05 \mathrm{M}$ Tris buffered saline (TBS) ( $\mathrm{pH} 7.6)$ with $0.1 \%$ Tween 20 . Endogenous peroxidase activity was abolished by applying on sections 3\% hydrogen peroxide and a commercial protein block (serum free) reagent (X 0909, DAKO) was used to avoid aspecific bindings of the primary antibodies. The primary antibodies were polyclonal, raised in rabbit and directed against: human neuronal nitric oxide synthase (nNOS) (AB-5380; Chemicon International Inc.), porcine vasoactive intestinal polypeptide (VIP) (20077, Incstar) and porcine chromogranin A ( $\mathrm{Cr} \mathrm{A}$ ) (20086, DiaSorin). They were applied for $30^{\prime}$ at r.t.. The structures considered for the evaluation of the results were neurons contained in the intestinal nerve plexuses positive for nNOS and VIP, and endocrine cells scattered in the mucosa of the bowel stained by the anti- $\mathrm{Cr} \mathrm{A}$ antibody. These latter were also defined neuroendocrine (NE) cells (Pearse 1977) or paraneurons (Fujita 1980). To their staining, years ago (Cecio et al. 1988), the authors applied the argyrophil method of Linder (1978), originally developed for the study of nerve structures. The ABC and EVS methods were applied following the description included in the respective ready-to-use kits (PK-6101, Vector Laboratories; K 4010, DAK0), while the MICA was performed as described originally (Mangham and Isaacson 1999). Diaminobenzidine (DAB) was utilized as chromogen. The sequences of the EVS and MICA methods were combined (Figure 1) as follows:

1. application of a primary antiserum for $30^{\prime}$ at r.t.,

2. incubation in the dextran polymer solution for $30^{\prime}$ at r.t. (the polymer binds goat anti-rabbit IgGs, as secondary antibodies, and peroxidases, as revealers),

3. incubation in a rabbit anti-goat (RAG) IgG solution ( $1: 800$ in TBS) for $20^{\prime}$ at r.t.,

4. incubation in a peroxidase-labeled goat anti-rabbit (GAR) IgG solution ( $1: 400$ in TBS) for $20^{\prime}$ at r.t.,

5. incubation in a RAG IgG solution ( $1: 800$ in TBS) for $20^{\prime}$ at r.t.,

6. incubation in a peroxidase-labeled GAR IgG solution ( $1: 400$ in TBS) for $20^{\prime}$ at r.t.,

7. staining in a peroxidase-DAB solution until the desired intensity is developed.

The sections were rinsed in tap water, dehydrated through serial alcohols, cleared in xylene and mounted in Histovitrex (45982, Carlo Erba). Each step was followed by a $5^{\prime}$ wash in TBS-Tween 20

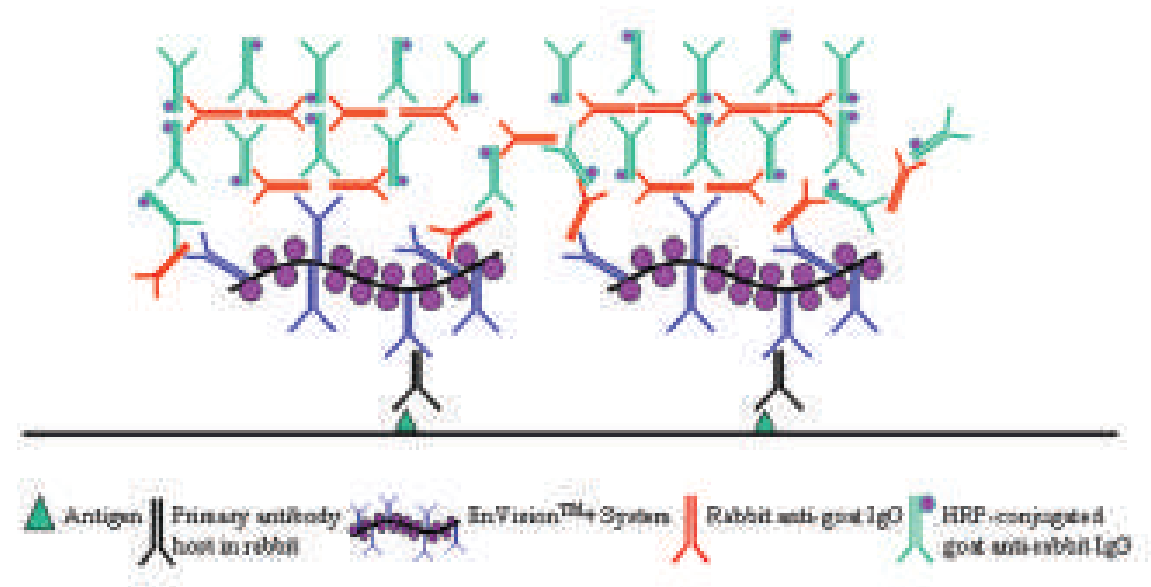

Figure 1. The combination of the sequences of EVS and MICA methods forms a molecular network whose sensitivity in revealing tissue antigens is four/eight times higher than those of the original methods. Schematic drawing. 


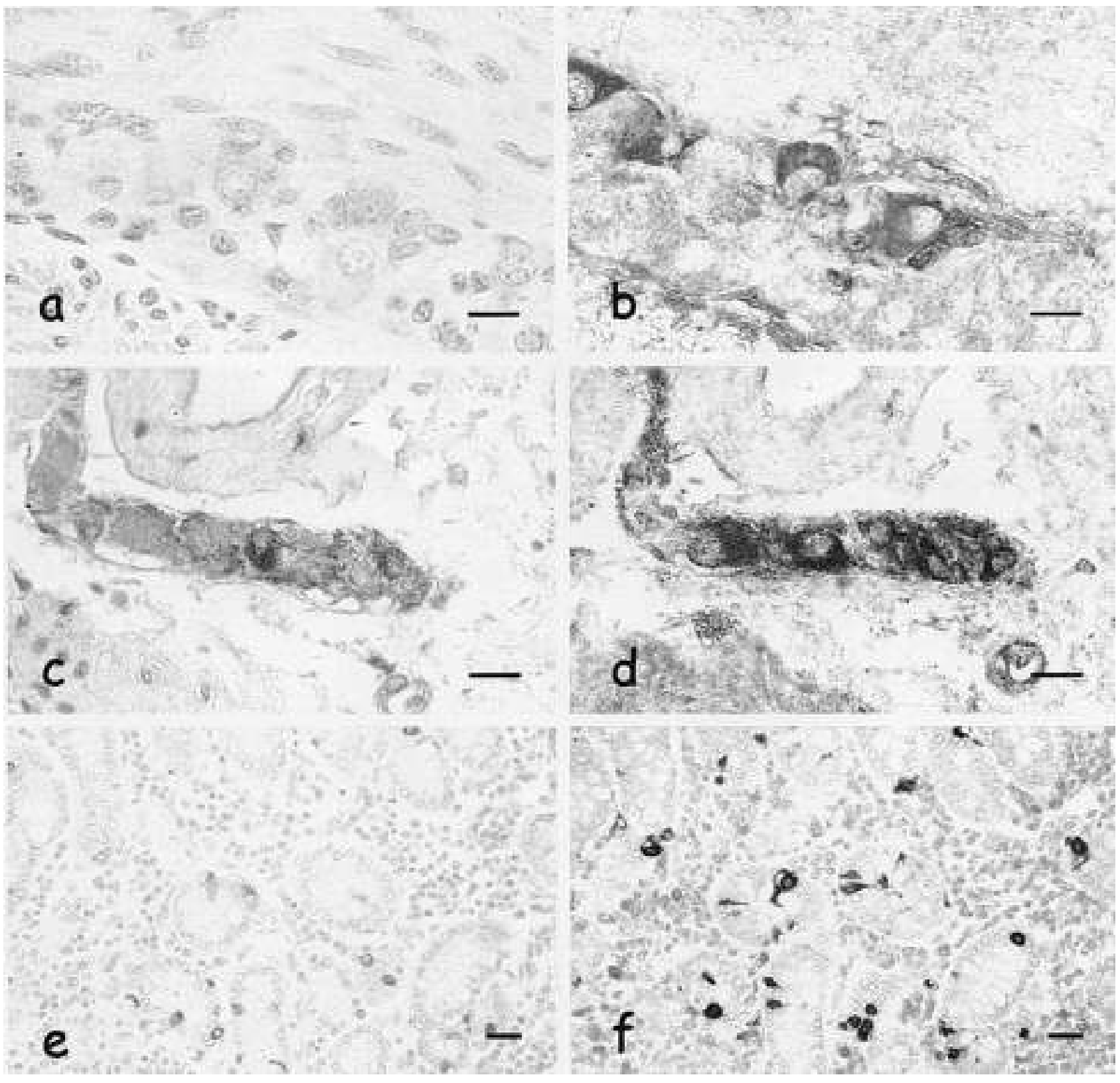

Figure 2 (a-f) - Three couples of consecutive sections stained by the EVS (a, c), MICA (e) and EVS/MICA (combined) (b, d, f) methods utilising primary antibodies directed against nNOS $(a, b), \operatorname{VIP}(c, d)$ and $\operatorname{Cr} A(e, f)$, respectively. a, b: at a dilution of 1:10,000 of the primary antiserum the EVS technique (a) is going to loose staining capability while the combined one (b) is perfectly active. c, d: the effectiveness of the combined method (d) is superior to those of the original not only at high but also at low-intermediate dilutions $(1: 4,000)$ of the primary antibody. e, $f$ : notwithstanding the high dilution of the anti-Cr A antibody $(1: 64,000)$ the combined method (f), here compared to the MICA one (e), clearly shows structural details as cytoplasmic projections. Figs. 2a, b: sheep; figs. 2c, d, e, f: pig. Figs. 2a, c, e, f: haematoxylin counterstaining. All bars: $20 \mu \mathrm{m}$.

buffer. The IgG dilutions used in the steps 3 and 5 and also 4 and 6 were chosen in a series ranging from $1: 300$ to $1: 1,500$ on the basis of the results observed. Sometimes counterstaining by haematoxylin was used.

The comparison between the four immunohistochemical techniques was performed as follows. The sections of a consecutive series were stained alter- nately by two methods using a single primary antibody. The dilution of this latter started from a minimum value, used for the first couple of sections, and was doubled for the successive couples up to the positivities disappeared. In this way the techniques were compared observing positive structures (often the same) contained in adjacent sections, for all values of the dilution scale. Such values ranged 
from $1: 2,500$ to $1: 320,000$ for the anti-nNOS antibody and from 1:1,000 to 1:512,000 for the anti-VIP and anti-Cr A antibodies. The staining intensity was evaluated independently by three investigators which adopted the following scale of values: negative $(-)$, very weak $( \pm)$, weak $(+)$, moderate $(++)$, strong $(+++)$ and very strong $(++++)$. The staining background was discussed without introducing a precise evaluation scheme.

Controls for the antibodies used were performed by substituting the primaries with normal rabbit serum or buffer, and the others with buffer alone. Controls were always negative.

The preparations were observed using a 600 Eclipse Nikon light microscope and microphotographs were taken using Technical Pan b/w Kodak film.

\section{Results}

In our hands the EVS and MICA methods resulted almost equivalent regarding sensitivity of detection and staining intensity, the first being slightly preferred. When compared to the routinely used $A B C$ staining, they were found to be roughly two times more sensitive. All techniques gave a fine, clear background but, in the case of $A B C$, this result was obtained only when a previous blockade of the endogenous biotins was performed.

The EVS/MICA (combined) method resulted much more sensitive than the techniques from which it was derived. It was able to evidentiate nervous and/or endocrine structures up to a primary antibody dilution value which was four-eight times greater than the highest still effective for the original stainings. Moreover the observation of couples of consecutive sections, the first stained by the
EVS (Figure 2a, c) or MICA (Figure 2e) methods and the second by the combined method (Figure $2 b$, $d, f$ ), showed that this latter was more effective for sensitivity and staining intensity, at both low and high dilutions. The combined method gave a background slightly higher than those of the original stainings but always compatible with an optimal view of the microscopic field.

The presence of RAG IgGs in the sequences of the combined and MICA methods caused enhancement of background when such techniques were performed on sheep tissues although the antisera diluent was added with normal sheep serum or sheep IgGs. This phenomenon, notoriously due to the presence of endogenous Igs cross reacting with the cited antibody, was moderate in the intestinal muscle and submucosa but somewhat higher in the mucosa. For this reason the evaluation of the positivity of mucosal NE cells was not considered in the sheep.

The results described above are summarized in the Tables 1, 2 and 3.

\section{Discussion}

As described, the EVS and MICA methods resulted more effective than the $A B C$ method regarding sensitivity of detection. This finding confirms analogous results recently obtained by others, which compared both methods, separately, to immunohistochemical techniques utilizing the avidin-biotin complex (Heras et al. 1995, Sabattini et al. 1998, Vyberg and Nielsen 1998, Mangham and Isaacson 1999, Mangham et al. 2000). Moreover, EVS is particularly rapid, consisting of two steps, while MICA is by far the less expensive. On this basis the adoption of the EVS and MICA methods in current

Table 1. Sensitivity of the immunohistochemical techniques studied in staining nNOS-containing nerve structures (cell bodies and fibres) of the pig and sheep small and large intestine.

\begin{tabular}{|c|c|c|c|c|c|c|c|c|}
\hline \multirow{3}{*}{ Dilutions } & \multicolumn{8}{|c|}{ Techniques and species } \\
\hline & \multicolumn{2}{|c|}{$A B C$} & \multicolumn{2}{|c|}{ EVS } & \multicolumn{2}{|c|}{ MICA } & \multicolumn{2}{|c|}{ EVS/MICA } \\
\hline & pig & sheep & pig & sheep & pig & sheep & pig & sheep \\
\hline $1: 2,500$ & + & + & ++ & +++ & $+/++$ & ++ & +++ & ++++ \\
\hline $1: 5,000$ & \pm & + & + & ++ & + & $+/++$ & ++ & ++++ \\
\hline $1: 10,000$ & - & \pm & - & + & - & + & $+/++$ & +++ \\
\hline $1: 20,000$ & & - & & - & & - & + & ++ \\
\hline $1: 40,000$ & & & & & & & \pm & $+/++$ \\
\hline $1: 80,000$ & & & & & & & - & + \\
\hline $1: 160,000$ & & & & & & & & \pm \\
\hline $1: 320,000$ & & & & & & & & - \\
\hline
\end{tabular}

- : negative; \pm : very weak; + : weak; ++ : moderate; +++ : strong; ++++ : very strong. 
Table 2. Sensitivity of the immunohistochemical techniques studied in staining VIP-containing nerve structures (cell bodies and fibres) of the pig and sheep small and large intestine.

\begin{tabular}{|c|c|c|c|c|c|c|c|c|}
\hline \multirow{3}{*}{ Dilutions } & \multicolumn{8}{|c|}{ Techniques and species } \\
\hline & \multicolumn{2}{|c|}{$A B C$} & \multicolumn{2}{|c|}{ EVS } & \multicolumn{2}{|c|}{ MICA } & \multicolumn{2}{|c|}{ EVS/MICA } \\
\hline & pig & sheep & pig & sheep & pig & sheep & pig & sheep \\
\hline $1: 1,000$ & $++/+++$ & +++ & +++ & +++ & +++ & +++ & ++++ & ++++ \\
\hline $1: 2,000$ & ++ & ++ & +++ & +++ & $++/+++$ & $++/+++$ & ++++ & ++++ \\
\hline $1: 4,000$ & + & + & ++ & +++ & +/++ & ++ & ++++ & ++++ \\
\hline $1: 8,000$ & \pm & + & + & ++ & + & $+/++$ & +++ & +++ \\
\hline $1: 16,000$ & - & \pm & - & + & - & $\pm /+$ & $++/+++$ & $++/+++$ \\
\hline $1: 32,000$ & & - & & - & & - & ++ & ++ \\
\hline $1: 64,000$ & & & & & & & $+/++$ & $+/++$ \\
\hline $1: 128,000$ & & & & & & & + & + \\
\hline $1: 256,000$ & & & & & & & \pm & \pm \\
\hline $1: 512,000$ & & & & & & & - & - \\
\hline
\end{tabular}

- : negative; \pm : very weak; + : weak; ++ : moderate; +++ : strong; ++++ : very strong.

Table 3. Sensitivity of the immunohistochemical techniques studied in staining $\mathrm{Cr}$ A-containing NE cells of the pig small and large intestine.

\begin{tabular}{lcccc}
\hline \hline \multicolumn{5}{c}{ Techniques } \\
Dilutions & ABC & EVS & MICA & EVS/MICA \\
& & & & \\
\hline $1: 1,000$ & ++++ & ++++ & ++++ & ++++ \\
$1: 2,000$ & ++++ & ++++ & ++++ & ++++ \\
$1: 4,000$ & +++ & +++ & +++ & ++++ \\
$1: 8,000$ & ++ & +++ & $++/+++$ & ++++ \\
$1: 16,000$ & ++++ & +++ & ++ & +++ \\
$1: 32,000$ & + & ++ & $+/++$ & +++ \\
$1: 64,000$ & - & + & $\pm /+$ & $++/+++$ \\
$1: 128,000$ & & \pm & \pm & ++ \\
$1: 256,000$ & & - & - & + \\
$1: 512,000$ & & & & - \\
\hline
\end{tabular}

- : negative; \pm : very weak; + : weak; ++ : moderate; +++ : strong; ++++ : very strong.

laboratory use may be advised, with a preference for the first.

The high sensitivity of the combined method can be explained by the great number of revealing molecules of peroxidases contained in its sequence and, hypothetically, by the following two considerations. Some of the GAR IgGs linked to the dextran molecules do not react with primary antibodies (Sabattini et al. 1998) and are bound, reciprocally, by the RAG IgGs adsorbed on the section in step 3 . These latter are bound, still reciprocally, by the peroxidase-labeled GAR IgGs used in step 4. This chain made by tight interactions of antibodies directed against each other could give rise to a network of IgGs firmly linked to the tissue antigens (Mangham et al. 2000). Moreover the repeated use of peroxidase-labeled GAR IgGs (steps 4 and 6) along the combined method is a warranty that at least the majority (if not all) of the tissue antigens are revealed whereas, in the EVS technique, part of them could remain unrevealed due to a possible steric obstruction caused by the big molecules of dextran.

The effectiveness of the combined method could be further enhanced by the following operations:

a) execution of all steps of the sequence at $37^{\circ} \mathrm{C}$,

b) use of peroxidase-labeled IgGs also in steps 3 and 5 ,

c) enhancement of the $D A B$ staining by using heavy metals (Adams 1981, Hsu and Soban 1982), imidazole (Straus 1982), silver (Gallyas et al. 1982, Merchenthaler et al. 1989) and the glucose oxidase-DAB-Nickel method (Ito et al. 1979, Shu et al. 1988),

d) overnight incubation of the primary antibody,

e) use of tiramide as signal amplifier (Adams 1992).

The operations listed in a) and b) and part of those listed in c) can be performed without lengthening the time of the immunostaining.

Obviously the cost of the combined method was higher than those of the original techniques but the aim of the enhancement of the immunohistochemical signal was achieved.

As far as sensitivity of revelation is concerned, the combined method worked well both on pig and sheep tissues so that interspecific differences were not seen.

In conclusion, we think that the combined method will find useful applications in several cases: detection of small quantities of tissue antigens, evidenti- 
ation of molecules partially masked by prolonged fixation/embedding procedures, use of expensive primary antibodies at high dilution and, finally, tracing of immunohistochemical pictures more complete than those given by the current routine techniques.

\section{References}

Adams JC. Heavy metal intensification of DAB-based HRP reaction product. J Histochem Cytochem 1981; 29:775.

Adams JC. Biotin amplification of biotin and horseradish peroxidase signal in histochemical stains. J Histochem Cytochem 1992; 40:1457-63.

Bisgaard K, Pulzek K. Use of polymer conjugates in immunohistochemistry: a comparative study of a traditional staining method to a staining method utilizing polymer conjugates. Pathol Int 1996; 46 (suppl. 1):577.

Cecio A, Vittoria A, Budetta G, Corona A. Staining of neuroendocrine cells by Linder's argyrophil method. J Anat 1988; 157:197-201.

Davidoff M, Schulze W. Combination of the peroxidase anti-peroxidase (PAP)- and avidin-biotin-peroxidase complex (ABC)-techniques:an amplification alternative in immunocytochemical staining. Histochem 1990; 93:531-6.

Fujita T. Paraneuron, its current implications. Biomed Res 1980; 1 (suppl.):3-9.

Gallyas F, Görcs T, Merchenthaler I. High-grade intensification of the end-product of the diaminobenzidine reaction for peroxidase histochemistry. J Histochem Cytochem 1982; 30:183-84.

Heras A, Roach CM, Key ME. Enhanced polymer detection system for immunohistochemistry. Mod Pathol 1995; 8:165A.

Hsu SM, Raine L, Fanger $\mathrm{H}$. Use of avidin-biotin-peroxidase complex $(A B C)$ in immunoperoxidase techniques: a comparison between $A B C$ and unlabeled antibody (PAP) procedures. J Histochem Cytochem $1981 ; 29: 577-80$.

Hsu SM, Soban E. Color modification of diaminobenzidine (DAB) precipitation by metallic ions and its application for double immuno- histochemistry. J Histochem Cytochem 1982; 30:1079-82.

Itoh K, Konishi A, Nomura S, Mizuno N, Nakamura $Y$, Sugimoto T. Application of coupled oxidation reaction to electron microscopic demonstration of horseradish peroxidase:cobalt-glucose oxidase method. Brain Res 1979; 175:341-46.

Linder JE. A simple and reliable method for the silver impregnation of nerves in paraffin sections of soft and mineralized tissues. $J$ Anat 1978; 127:543-51.

Mangham DC, Isaacson PG. A novel immunohistochemical detection system using mirror image complementary antibodies (MICA). Histopathol 1999; 35:129-33.

Mangham DC, Bradwell AR, Isaacson PG. MICA-a highly sensitive and avidin-free immunohistochemical detection system. Adv Anat Pathol $2000 ; 7: 360-4$.

Merchenthaler I, Stankovics J, Gallyas F. A highly sensitive one-step method for silver intensification of the nickel-diaminobenzidine endproduct of peroxidase reaction. J Histochem Cytochem 1989; 37:1563-5.

Pearse AGE. The diffuse neuroendocrine system and the APUD concept:related "endocrine" peptides in brain, intestine, pituitary, placenta and anuran cutaneous glands. Med Biol 1977; 55:115-25.

Sabattini E, Bisgaard K, Ascani S, Poggi S, Piccioli M, Ceccarelli C, et al. The EnVisionTM+ System:a new immunohistochemical method for diagnostic and research. Critical comparison with the APAAP, ChemMate, CSA, LABC, and SABC techniques. J Clin Pathol 1998; 51:506-11.

Shu S, Ju G, Fan L. The glucose oxidase-DAB-nickel method in peroxidase histochemistry of the nervous system. Neurosci Lett 1988; 85:169-71

Straus W. Imidazole increase the sensitivity of cytochemical reaction for peroxidase with diaminobenzidine at a neutral $\mathrm{pH}$. J Histochem Cytochem 1982; 30:491-3.

Swanson PE, Hagen KA, Wick MR. Avidin-biotin-peroxidase-antiperoxidase (ABPAP) complex. An immunocytochemical method with enhanced sensitivity. Am J Clin Pathol 1987; 88:162-76.

Vacca LL, Rosario S, Zimmerman EA, Tomachefsky P, Po-Ying NG, Hsu KC. Application of immunoperoxidase techniques to localize horseradish peroxidase-tracer in the central nervous system. J Histochem Cytochem 1975; 23:208-15.

Vyberg M, Nielsen S. Dextran polymer conjugate two-step visualization system for immunohistochemistry. A comparison of Envision+ with two three step avidin-biotin techniques. Appl Immunohistochem 1998; 6:3-10. 